\title{
Visualizing Influential \\ Observations in Dependent Data
}

\author{
MARC G. GENTON AND ANNE RUIZ-GAZEN
}




\title{
Visualizing Influential Observations in Dependent Data
}

\author{
Marc G. Genton ${ }^{1}$ and Anne Ruiz-Gazen ${ }^{2}$
}

June 23, 2009

\begin{abstract}
We introduce the hair-plot to visualize influential observations in dependent data. It consists of all trajectories of the value of an estimator when each observation is modified in turn by an additive perturbation. We define two measures of influence: the local influence which describes the rate of departure from the original estimate due to a small perturbation of each observation; and the asymptotic influence which indicates the influence on the original estimate of the most extreme contamination for each observation. The cases of estimators defined as quadratic forms or ratios of quadratic forms are investigated in detail. Sample autocovariances, covariograms and variograms belong to the first case. Sample autocorrelations, correlograms, and indices of spatial autocorrelation such as Moran's $I$ belong to the second case. We illustrate our approach on various datasets from time series analysis and spatial statistics.
\end{abstract}

Some key words: Autocovariance; Moran's I; Outlier; Quadratic form; Robustness; Space; Time; Variogram.

Short title: Visualizing Influential Observations

\footnotetext{
${ }^{1}$ Department of Statistics, Texas A\&M University, College Station, TX 77843-3143, USA. E-mail: genton@stat.tamu.edu Genton's research was supported in part by NSF grants DMS-0504896, CMG ATM-0620624, and by Award No. KUS-C1-016-04, made by King Abdullah University of Science and Technology (KAUST).

${ }^{2}$ Toulouse School of Economics, Université des Sciences Sociales, 21 Allée de Brienne, 31000 Toulouse, France. E-mail: ruiz@cict.fr

The authors thank Thibault Laurent for implementing the hair-plot function in R.
} 


\section{Introduction}

Consider an estimator $\widehat{\theta}(\mathbf{Z})$ of a parameter $\theta$ based on a data vector $\mathbf{Z}=\left(Z_{1}, \ldots, Z_{n}\right)^{\mathrm{T}}$. In order to study influential observations on this estimator, we define a perturbation of $\mathbf{Z}$ by $\mathbf{Z}[i, \zeta]=\mathbf{Z}+\zeta \boldsymbol{e}_{i}$, where $\boldsymbol{e}_{i}$ has a nonzero component only at index $i$, at which it is 1 , and $\zeta$ represents the value of the perturbation. The effect of influential observations can be visualized with a plot of each $\widehat{\theta}(\mathbf{Z}[i, \zeta]), i=1, \ldots, n$, as a function of $\zeta$. We coin such a graphical representation a hair-plot. A hair-plot is a version of the empirical influence function with replacement (see Hampel et al., 1986, page 93) and with a particular parameterization of the perturbation. The perturbation $\zeta$ is added to the original sample so that at $\zeta=0$, the original value $\widehat{\theta}(\mathbf{Z})$ is recovered for any $i=1, \ldots, n$. Typically $\zeta$ is a real number but for certain applications, such as for a positive variable, the range of $\zeta$ can be restricted to ensure sensible values of the variable of interest. Note that in the case of dependent data, the version of the empirical influence function with replacement of an observation is preferable to the one with addition of an observation. Indeed, in the context of time series or spatial data, there is no obvious way to define a new instant or a new location of observation. This is in contrast to the classical case of independent and identically distributed (i.i.d.) observations where such an issue does not arise.

Associated to the hair-plot, two influential measures are then of interest. First, we define the local influence of the $i$-th observation on the estimator $\widehat{\theta}(\mathbf{Z})$ as

$$
\tau_{i}(\widehat{\theta}, \mathbf{Z})=\left.\frac{\partial}{\partial \zeta} \widehat{\theta}(\mathbf{Z}[i, \zeta])\right|_{\zeta=0}
$$

It describes the rate of departure from the value $\widehat{\theta}(\mathbf{Z})$ for each observation due to a small perturbation. Hence, the most influential observations correspond to the largest absolute values of $\tau_{i}(\widehat{\theta}, \mathbf{Z})$. Second, we define the asymptotic influence of the $i$-th observation on the 
estimator $\widehat{\theta}(\mathbf{Z})$ as

$$
\nu_{i}(\widehat{\theta}, \mathbf{Z})=\lim _{\zeta \rightarrow \infty} \widehat{\theta}(\mathbf{Z}[i, \zeta]) .
$$

It indicates the influence on the value $\widehat{\theta}(\mathbf{Z})$ of the most extreme contamination for each observation. Note that in general the function $\widehat{\theta}(\mathbf{Z}[i, \zeta])$ of $\zeta$ depends on $i$, the exception being the case where $\widehat{\theta}$ is the sample mean or a function of it, as we show next.

Proposition 1. Let $f$ be a function from $\mathbb{R}^{n}$ into $\mathbb{R}$ such that for any $z_{1}, z_{2}, \ldots, z_{n}$ in $\mathbb{R}$, any $i, j=1, \ldots, n$ and $\zeta$ in $\mathbb{R}$,

$$
f\left(z_{1}, \ldots, z_{i-1}, z_{i}+\zeta, z_{i+1}, \ldots, z_{j}, \ldots, z_{n}\right)=f\left(z_{1}, \ldots, z_{i}, \ldots, z_{j-1}, z_{j}+\zeta, z_{j+1}, \ldots, z_{n}\right) .
$$

Then $f$ is a function of $\sum_{i=1}^{n} z_{i}$.

The proof of this result is given in the Appendix.

We have implemented a command hair.plot (data, thetahat,...) in $R$ ( $R$ Development Core Team, 2009) that is available from the authors upon request. It produces a hair-plot and allows to identify each observation (i.e., each hair) in the plot. For illustration, we consider a dataset of $n=91$ monthly interest rates of an Austrian bank. Künsch (1984), Ma and Genton (2000), Azzalini and Genton (2008), and Wang et al. (2009) have previously studied this dataset in the context of robust time series analysis and noted the presence of three large outliers for the months 18, 28 and 29. Because these authors have argued that an autoregressive model of order one, $\mathrm{AR}(1)$, is appropriate for these data, we focus on the lag-one sample autocorrelation $\widehat{r}(1)=0.78$ obtained from

$$
\widehat{r}(h)=\frac{\sum_{i=1}^{n-h}\left(Z_{i+h}-\bar{Z}\right)\left(Z_{i}-\bar{Z}\right)}{\sum_{i=1}^{n}\left(Z_{i}-\bar{Z}\right)^{2}}, \quad 0 \leq h \leq n-1,
$$

where $\bar{Z}$ is the sample mean. The top panel of Figure 1 depicts the hair-plot of $\widehat{r}(1)$ for $\zeta \in[-3,3]$ and allows to identify any curve, that is, any observation. It reveals that the months 17, 18, 19 and 27, 30 are quite influential. The months 76 is also identified to be somewhat influential. 

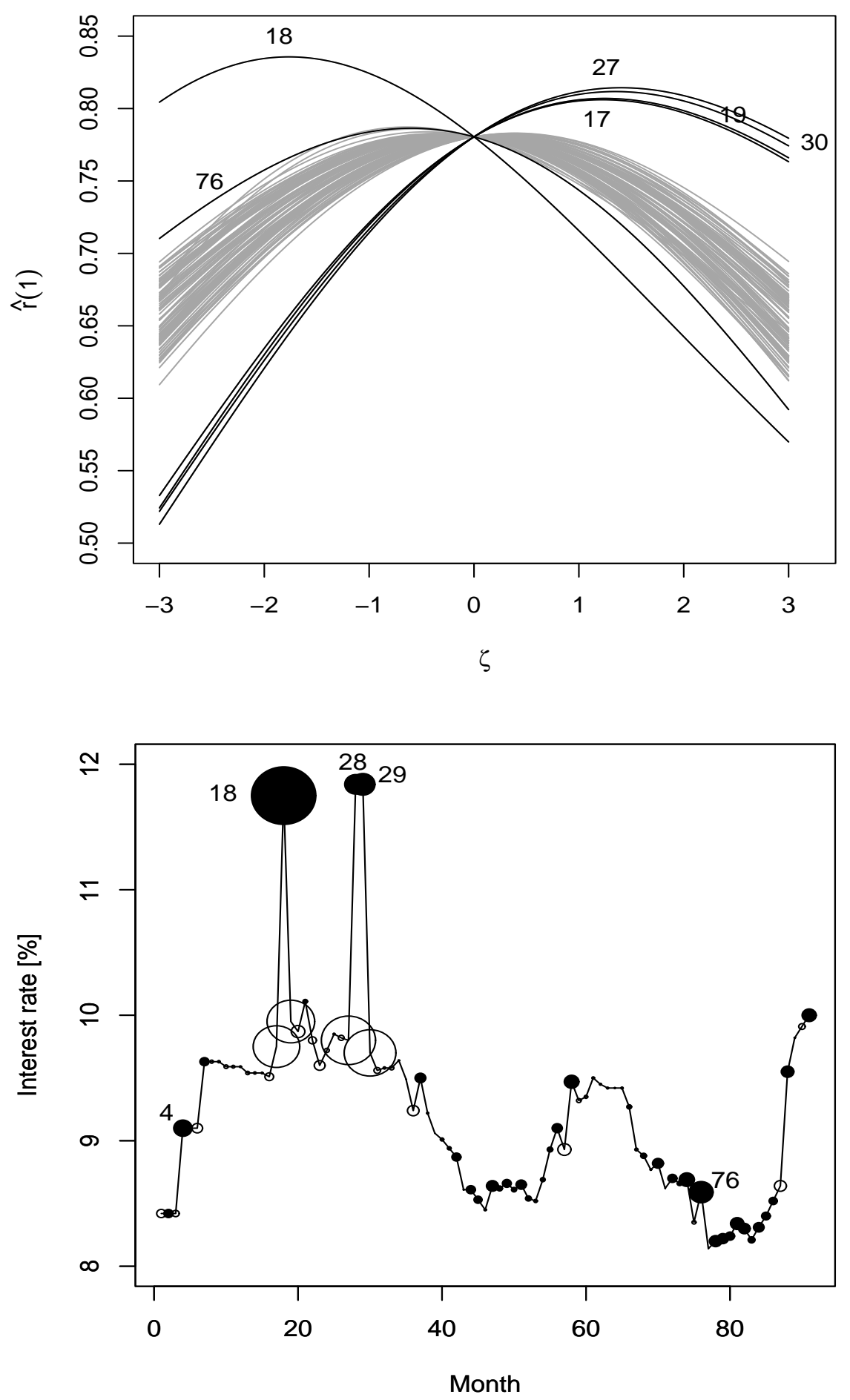

Figure 1: Hair-plot and disc-plot (for $\zeta>0$ ) of the lag-one sample autocorrelation $\widehat{r}(1)$ on the monthly interest rates dataset. 
The bottom panel of Figure 1 presents a disc-plot for $\zeta>0$ of the monthly interest rates time series. The radii of the discs are proportional to the rate of departure from $\widehat{r}(1)=0.78$ due to a small contamination at each observation. Open discs denote an increase of the value $\widehat{r}(1)$ whereas closed discs denote a decrease. The discs with large radii correspond to the most influential observations. The months 4 and 76 are also seen to be somewhat influential.

The paper is organized as follows. In Section 2, we study the local and asymptotic influence of estimators that are defined as quadratic forms in the data vector, whereas we concentrate on ratios of quadratic forms in Section 3. Sample autocovariances, covariograms and variograms belong to the former. Sample autocorrelations, correlograms, and indices of spatial autocorrelation such as Moran's I belong to the latter. In Section 4, we illustrate our approach on applications to pollution data and to African conflict data. We end with a discussion in Section 5 where we propose the use of the hair-plot on robust estimators for dependent data. We also discuss extensions to the case of multiple simultaneous influential observations.

\section{Influence on Quadratic Forms}

We study the effect of an influential observation on estimators defined as quadratic forms in the data vector, that is, $\widehat{\theta}_{Q}(\mathbf{Z}, \mathbf{A})=\mathbf{Z}^{\mathrm{T}} \mathbf{A} \mathbf{Z}$, where $\mathbf{A}=\left(a_{i j}\right)$ is an $n \times n$ matrix. It follows that, under the contamination scheme $\mathbf{Z}[i, \zeta]$, we have:

$$
\widehat{\theta}_{Q}(\mathbf{Z}[i, \zeta], \mathbf{A})=\mathbf{Z}^{\mathrm{T}} \mathbf{A} \mathbf{Z}+\left(\mathbf{Z}^{\mathrm{T}} \mathbf{A} \boldsymbol{e}_{i}+\boldsymbol{e}_{i}^{\mathrm{T}} \mathbf{A} \mathbf{Z}\right) \zeta+\left(\boldsymbol{e}_{i}^{\mathrm{T}} \mathbf{A} \boldsymbol{e}_{i}\right) \zeta^{2}
$$

Therefore, the local and asymptotic influences (1) and (2) of the $i$-th observation are respectively:

$$
\begin{aligned}
\tau_{i}\left(\widehat{\theta}_{Q}, \mathbf{Z}\right) & =\mathbf{Z}^{\mathrm{T}} \mathbf{A} \boldsymbol{e}_{i}+\boldsymbol{e}_{i}^{\mathrm{T}} \mathbf{A} \mathbf{Z}, \\
\nu_{i}\left(\widehat{\theta}_{Q}, \mathbf{Z}\right) & =\infty .
\end{aligned}
$$


If the matrix $\mathbf{A}$ is symmetric, then the local influence reduces to $\tau_{i}\left(\widehat{\theta}_{Q}, \mathbf{Z}\right)=2 \mathbf{a}_{i}^{\mathrm{T}} \mathbf{Z}$ where $\mathbf{a}_{i}$ is the $i$-th row of the matrix $\mathbf{A}$.

In time series analysis, the sample autocovariance function is an important tool defined at time lag $h$ by

$$
\widehat{c}(h)=\frac{1}{n} \sum_{i=1}^{n-h}\left(Z_{i+h}-\bar{Z}\right)\left(Z_{i}-\bar{Z}\right), \quad 0 \leq h \leq n-1 .
$$

It can also be expressed as a quadratic form in the vector of time series data. The corresponding matrix is $\mathbf{A}=\frac{1}{n} \mathbf{H D}(h) \mathbf{H}$ where $\mathbf{H}=\mathbf{I}_{n}-\frac{1}{n} \mathbf{1}_{n} \mathbf{1}_{n}^{\mathrm{T}}$ is the symmetric and idempotent $\left(\mathbf{H}^{2}=\mathbf{H}\right)$ centering matrix with $\mathbf{I}_{n}$ the $n \times n$ identity matrix and $\mathbf{1}_{n}$ the $n$-vector of ones. The matrix $\mathbf{D}(h)=\frac{1}{2}\left(\mathbf{P}(h)+\mathbf{P}(h)^{\mathrm{T}}\right)$ is the temporal design matrix at lag $h$, where $\mathbf{P}(h)$ is an $n \times n$ matrix with ones on the $h$-th upper diagonal and zeroes elsewhere, $1 \leq h \leq n-1$, and $\mathbf{P}(0)=\mathbf{I}_{n}$, see Genton (1999). Therefore, the matrix $\mathbf{A}$ is symmetric and the local influence of the $i$-th observation on the sample autocovariance function takes the form $\tau_{i}(\widehat{c}(h), \mathbf{Z})=2 \mathbf{a}_{i}^{\mathrm{T}} \mathbf{Z}$ with the $j$-th component of the vector $\mathbf{a}_{i}$ given by:

$$
a_{i j}=\frac{1}{n}\left\{d_{i j}(h)-\frac{1}{n} d_{i \cdot}(h)-\frac{1}{n} d_{\cdot j}(h)+\frac{1}{n^{2}} d_{. .}(h)\right\}
$$

where $\mathbf{D}(h)=\left(d_{i j}(h)\right)$ with $d_{i .}(h)=\sum_{j=1}^{n} d_{i j}(h), d_{. j}(h)=\sum_{i=1}^{n} d_{i j}(h)$, and $d_{. .}(h)=$ $\sum_{i=1}^{n} \sum_{j=1}^{n} d_{i j}(h)$. Note that the local influence changes as a function of the temporal lag $h$. From the particular form of the matrix $\mathbf{D}(h)$, it follows that $d . .(h)=n-h$. Moreover, if $1 \leq h \leq\left\lfloor\frac{n}{2}\right\rfloor$ then $d_{i \cdot}(h)=d_{\cdot i}(h)=1$ for $h<i<n-h$ and $d_{i \cdot}(h)=d_{\cdot i}(h)=\frac{1}{2}$ otherwise. If $\left\lfloor\frac{n}{2}\right\rfloor<h \leq n-1$ then $d_{i .}(h)=d_{. i}(h)=0$ for $h<i<n-h$ and $d_{i \cdot}(h)=d_{\cdot i}(h)=1$ otherwise.

Similarly in spatial analysis, the sample covariogram is defined by

$$
\widehat{c}(\mathbf{h})=\frac{1}{n} \sum_{(i, j) \in N_{\mathbf{h}}}\left(Z_{i}-\bar{Z}\right)\left(Z_{j}-\bar{Z}\right),
$$

where $N_{\mathbf{h}}$ is the set of spatial locations separated by the lag vector $\mathbf{h}$. It can be rewritten as a quadratic form in the vector of data with $\mathbf{A}=\frac{1}{n} \mathbf{H D}(\mathbf{h}) \mathbf{H}$ where now $\mathbf{h}$ is a lag vector 
in space. Thus, the description of the matrix $\mathbf{D}(\mathbf{h})$ is more involved, but (4) still holds and expressions for its terms can be derived. The sample variogram is an alternative tool for measuring spatial dependence and is defined by:

$$
2 \widehat{\gamma}(\mathbf{h})=\frac{1}{\left|N_{\mathbf{h}}\right|} \sum_{(i, j) \in N_{\mathbf{h}}}\left(Z_{i}-Z_{j}\right)^{2},
$$

where $\left|N_{\mathbf{h}}\right|$ is the cardinality of the set $N_{\mathbf{h}}$. It can also be defined as a quadratic form in the vector of data with $\mathbf{A}=\mathbf{D}^{*}(\mathbf{h})$. Here, the specific form of $\mathbf{D}^{*}(\mathbf{h})$ is different from the one of $\mathbf{D}(\mathbf{h})$, see Genton (1998a), Gorsich et al. (2002), and Hillier and Martellosio (2006). The local influence for the sample variogram is still given by $(3)$ with $\mathbf{D}(h)$ replaced by $\mathbf{D}^{*}(\mathbf{h})$ in (4).

\section{Influence on Ratios of Quadratic Forms}

We investigate the effect of an influential observation on estimators defined as a ratio of two quadratic forms, for example such as the lag-one autocorrelation studied in the introduction. Traditionally, the asymptotic influence of an observation has been to push the value of the estimator to the edge of the parameter space. However, Genton and Lucas (2003, 2005) and Genton (2003) have shown that this needs not be the case for time series and spatial statistics settings. Instead, the estimator is sometimes pushed towards the center of the parameter space by a single outlying value. In Figure 1 we have seen the behavior of the lag-one autocorrelation for perturbations $\zeta$ within the interval $[-3,3]$. How will an estimator defined as a ratio of two quadratic forms based on the contaminated sample $\mathbf{Z}[i, \zeta]$ be affected when $\zeta$ becomes large?

To this end, we investigate the behavior of an estimator defined by

$$
\widehat{\theta}_{R Q}(\mathbf{Z}, \mathbf{A}, \mathbf{B})=\frac{\mathbf{Z}^{\mathrm{T}} \mathbf{A} \mathbf{Z}}{\mathbf{Z}^{\mathrm{T}} \mathbf{B Z}}
$$


where $\mathbf{A}=\left(a_{i j}\right)$ and $\mathbf{B}=\left(b_{i j}\right)$ are $n \times n$ matrices, under the contamination scheme $\mathbf{Z}[i, \zeta]$. It follows that:

$$
\widehat{\theta}_{R Q}(\mathbf{Z}[i, \zeta], \mathbf{A}, \mathbf{B})=\frac{\mathbf{Z}^{\mathrm{T}} \mathbf{A} \mathbf{Z}+\left(\mathbf{Z}^{\mathrm{T}} \mathbf{A} \boldsymbol{e}_{i}+\boldsymbol{e}_{i}^{\mathrm{T}} \mathbf{A} \mathbf{Z}\right) \zeta+\left(\boldsymbol{e}_{i}^{\mathrm{T}} \mathbf{A} \boldsymbol{e}_{i}\right) \zeta^{2}}{\mathbf{Z}^{\mathrm{T}} \mathbf{B Z}+\left(\mathbf{Z}^{\mathrm{T}} \mathbf{B} \boldsymbol{e}_{i}+\boldsymbol{e}_{i}^{\mathrm{T}} \mathbf{B Z}\right) \zeta+\left(\boldsymbol{e}_{i}^{\mathrm{T}} \mathbf{B} \boldsymbol{e}_{i}\right) \zeta^{2}}
$$

Therefore, the local and asymptotic influence of the $i$-th observation are respectively:

$$
\begin{aligned}
\tau_{i}\left(\widehat{\theta}_{R Q}, \mathbf{Z}\right) & =\frac{\left(\mathbf{Z}^{\mathrm{T}} \mathbf{A} \boldsymbol{e}_{i}+\boldsymbol{e}_{i}^{\mathrm{T}} \mathbf{A} \mathbf{Z}\right)\left(\mathbf{Z}^{\mathrm{T}} \mathbf{B Z}\right)-\left(\mathbf{Z}^{\mathrm{T}} \mathbf{A} \mathbf{Z}\right)\left(\mathbf{Z}^{\mathrm{T}} \mathbf{B} \boldsymbol{e}_{i}+\boldsymbol{e}_{i}^{\mathrm{T}} \mathbf{B Z}\right)}{\left(\mathbf{Z}^{\mathrm{T}} \mathbf{B Z}\right)^{2}}, \\
\nu_{i}\left(\widehat{\theta}_{R Q}, \mathbf{Z}\right) & =\frac{a_{i i}}{b_{i i}},
\end{aligned}
$$

provided that $b_{i i} \neq 0$. Hence, the asymptotic influence on the estimator is dictated by the ratio of the $i i$-th entries of the matrices $\mathbf{A}$ and $\mathbf{B}$. If the matrices $\mathbf{A}$ and $\mathbf{B}$ are symmetric, then the local influence reduces to

$$
\tau_{i}\left(\widehat{\theta}_{R Q}, \mathbf{Z}\right)=\frac{2}{\mathbf{Z}^{\mathrm{T}} \mathbf{B Z}}\left(\mathbf{a}_{i}-\widehat{\theta}_{R Q} \mathbf{b}_{i}\right)^{\mathrm{T}} \mathbf{Z}
$$

where $\mathbf{a}_{i}$ and $\mathbf{b}_{i}$ are the $i$-th rows of the matrices $\mathbf{A}$ and $\mathbf{B}$, respectively. We study these quantities for various estimators below.

Returning to time series analysis, the sample autocorrelation function studied in the introduction can be written as a ratio of quadratic forms in the data vector with $\mathbf{A}=$ $\mathbf{H D}(h) \mathbf{H}$ and $\mathbf{B}=\mathbf{H}$, where $\mathbf{H}$ and $\mathbf{D}(h)$ were defined in Section 2. Hence, its local influence is given by (5) where $\mathbf{a}_{i}$ and $\mathbf{b}_{i}$ can be derived based on (4). Its asymptotic influence is simply given by:

$$
\nu_{i}(\widehat{r}(h), \mathbf{Z})=\frac{n-h-2 n d_{i \cdot}(h)}{n(n-1)},
$$

where $d_{i}(h)$ is defined after (4). Note that $\lim _{n \rightarrow \infty} \nu_{i}(\widehat{r}(h), \mathbf{Z})=0$ for any observation $i$. Similarly in spatial analysis, the sample correlogram can be written as a quadratic form in the vector of data with $\mathbf{A}=\mathbf{H D}(\mathbf{h}) \mathbf{H}$ and $\mathbf{B}=\mathbf{H}$, where now $\mathbf{h}$ is a lag vector in space.

The presence of spatial dependence in data on a lattice is often assessed by means of a statistic such as Moran's $I$ (Moran, 1950). If $\mathbf{Z}=\left(Z_{1}, \ldots, Z_{n}\right)^{\mathrm{T}}$ is a spatial sample of 
dimension $n$, that is $Z_{i}$ represents an observation at the location $i$ on the lattice, then Moran's $I$ is defined by

$$
\widehat{I}(\mathbf{Z})=\frac{\sum_{i=1}^{n} \sum_{j=1}^{n} w_{i j}\left(Z_{i}-\bar{Z}\right)\left(Z_{j}-\bar{Z}\right)}{\sum_{i=1}^{n}\left(Z_{i}-\bar{Z}\right)^{2}},
$$

where the spatial structure matrix $\mathbf{W}=\left(w_{i j}\right)$ is of dimension $n \times n$ and contains nonnegative weights describing the degree of interaction between neighbor locations in the plane. The spatial structure matrix $\mathbf{W}$ does not need to be symmetric but can be transformed to symmetry by $\left(\mathbf{W}+\mathbf{W}^{T}\right) / 2$ without changing the value of Moran's $I$ defined in (7). Usually, the matrix $\mathbf{W}$ is obtained from a particular coding scheme in order to stabilize the heterogeneity resulting from different degrees of interaction between the spatial observations. In particular, one can distinguish between the globally standardized $C$-coding scheme, the row-sum standardized $W$-coding scheme, and the variance stabilizing $S$-coding scheme, see, e.g., Tiefelsdorf (2000). For all three coding schemes, the resulting matrix $\mathbf{W}$ is such that the sum of its elements is equal to $n$. The $W$-coding scheme is often used in practice and implies that $\mathbf{W} \mathbf{1}_{n}=\mathbf{1}_{n}$. Under normality of $\mathbf{Z}$, the expectation of Moran's $I$ under the hypothesis of independence is given by $\mathrm{E}(\widehat{I}(\mathbf{Z}))=-1 /(n-1)$, see, e.g., Cliff and Ord (1981, p. 44).

For the case of Moran's I defined in (7), we have again a ratio of quadratic forms in the data vector with $\mathbf{A}=\mathbf{H W H}$ and $\mathbf{B}=\mathbf{H}$. It follows that the local influence on Moran's $I$ can be derived similarly to the correlogram above. Regarding the asymptotic influence, we have $a_{i i}=\frac{1}{n}\left(w_{. .} / n-w_{\cdot i}-w_{i}\right)$ and $b_{i i}=1-1 / n$, where $w_{. .}=\sum_{i=1}^{n} \sum_{j=1}^{n} w_{i j}, w_{\cdot i}=\sum_{j=1}^{n} w_{j i}$, and $w_{i} .=\sum_{j=1}^{n} w_{i j}$. Hence

$$
\nu_{i}(\widehat{I}, \mathbf{Z})=\frac{1}{n-1}\left(w_{. .} / n-w_{\cdot i}-w_{i}\right)
$$

Consequently, as $\zeta \rightarrow \infty$, we have $\widehat{I}(\mathbf{Z}[i, \zeta]) \rightarrow \nu_{i}(\widehat{I}, \mathbf{Z})$ whatever the true value of $I$, i.e., whatever the realization of the uncontaminated sample Z. Moran's I no longer conveys any 
useful information on $I$ and the estimate is totally dictated by the contamination. Therefore, according to Genton and Lucas $(2003,2005)$, the breakdown-point of Moran's $I$ defined in (7) is zero. The precise value of the asymptotic influence $\nu_{i}(\widehat{I}, \mathbf{Z})$ given by (8) depends on the coding scheme. For all three coding schemes listed above, we have $w . .=n$. By noticing that $0 \leq w_{\cdot i}+w_{i} \leq n$, we obtain for the $C$-coding and $S$-coding schemes that $-1 \leq \nu_{i}(\widehat{I}, \mathbf{Z}) \leq 1 /(n-1)$, whereas for the $W$-coding scheme we have $-1 \leq \nu_{i}(\widehat{I}, \mathbf{Z}) \leq 0$. In the latter case, $\nu_{i}(\widehat{I}, \mathbf{Z})=-w_{\cdot i} /(n-1)$. If the observations are collected on a regular grid and the edge effects are neglected, then approximately we have $w_{\cdot i}=1$. In this case, Moran's $I$ estimator breaks down to $-1 /(n-1)$, the expected value of Moran's $I$ under the null hypothesis of independence, with one extreme contamination. Note that $-1 /(n-1)$ is not at the edge of the parameter space. In addition, the aforementioned behavior of Moran's $I$ for large sample size $n$ will break the estimator towards 0 . For finite and infinite $\zeta$, there is dependence on $\mathbf{W}$ since $\zeta$ will affect several local averages depending on the structure of $\mathbf{W}$. Finally, note that when $\bar{Z}=0$, we have $\mathbf{A}=\mathbf{W}$ and $\mathbf{B}=\mathbf{I}_{n}$ for Moran's $I$, hence $a_{i i}=w_{i i}=0$ and $b_{i i}=1$. Therefore, $\nu_{i}(\widehat{I}, \mathbf{Z})=0$ in that case for any $n$.

Li et al. (2007) have put forward an alternative closed-form measure of spatial autocorrelation defined as an approximate profile likelihood estimator (APLE) of the spatial dependence parameter of a spatial autoregressive (SAR) model. The estimator, a ratio of two quadratic forms, is defined by

$$
\widehat{A P L E}(\mathbf{Z})=\frac{\mathbf{Z}^{\mathrm{T}} \mathbf{H}\left[\left(\mathbf{W}+\mathbf{W}^{\mathrm{T}}\right) / 2\right] \mathbf{H Z}}{\mathbf{Z}^{\mathrm{T}} \mathbf{H}\left[\mathbf{W}^{\mathrm{T}} \mathbf{W}+\operatorname{tr}\left(\mathbf{W}^{2}\right) \mathbf{I}_{n} / n\right] \mathbf{H Z}},
$$

where $\operatorname{tr}(\cdot)$ is the trace operator. Note that $(9)$ is a slight extension of the original definition of Li et al. (2007) who set $\bar{Z}=0$. We claim also that their use of $\boldsymbol{\lambda}^{\mathrm{T}} \boldsymbol{\lambda}$, where $\boldsymbol{\lambda}$ is the explicit vector of eigenvalues of $\mathbf{W}$, is unnecessary since $\boldsymbol{\lambda}^{\mathrm{T}} \boldsymbol{\lambda}=\operatorname{tr}\left(\mathbf{W}^{2}\right)$. For the case of APLE defined in (9), we have $\mathbf{A}=\mathbf{H}\left[\left(\mathbf{W}+\mathbf{W}^{\mathrm{T}}\right) / 2\right] \mathbf{H}$ and $\mathbf{B}=\mathbf{H}\left[\mathbf{W}^{\mathrm{T}} \mathbf{W}+\operatorname{tr}\left(\mathbf{W}^{2}\right) \mathbf{I}_{n} / n\right] \mathbf{H}$, where again $\mathbf{H}$ is the centering matrix. Therefore, $a_{i i}=\frac{1}{n}\left(w_{. .} / n-w_{\cdot i}-w_{i}\right)$ and $b_{i i}=$ 


$$
\begin{aligned}
& \sum_{k} w_{k i}^{2}+\operatorname{tr}\left(\mathbf{W}^{2}\right)(n-1) / n^{2}-4\left(\sum_{k} w_{k i} w_{k}\right) / n-2\left(\sum_{k} w_{k}^{2}\right) / n^{2} . \text { Hence } \\
& \nu_{i}(\widehat{A P L E}, \mathbf{Z})=\frac{w_{. .} / n-w_{\cdot i}-w_{i} .}{n \sum_{k} w_{k i}^{2}+\operatorname{tr}\left(\mathbf{W}^{2}\right)(n-1) / n-4\left(\sum_{k} w_{k i} w_{k}\right)-2\left(\sum_{k} w_{k .}^{2}\right) / n} .
\end{aligned}
$$

Here again, as $\zeta \rightarrow \infty$, we have $\widehat{A P L E}(\mathbf{Z}[i, \zeta]) \rightarrow \nu_{i}(\widehat{A P L E}, \mathbf{Z})$ whatever the true value of $A P L E$, i.e., whatever the realization of the uncontaminated sample $\mathbf{Z}$. Therefore, according to Genton and Lucas $(2003,2005)$, the breakdown-point of the APLE statistic defined in

(9) is zero. It is more difficult to give bounds for $\nu_{i}(\widehat{A P L E}, \mathbf{Z})$ depending on the coding scheme of $\mathbf{W}$, but clearly for large $n$ we have $\nu_{i}(\widehat{A P L E}, \mathbf{Z}) \rightarrow 0$. When $\bar{Z}=0$, we have $\mathbf{A}=\left(\mathbf{W}+\mathbf{W}^{\mathrm{T}}\right) / 2$ and $\mathbf{B}=\mathbf{W}^{\mathrm{T}} \mathbf{W}+\operatorname{tr}\left(\mathbf{W}^{2}\right) \mathbf{I}_{n} / n$ for the APLE statistic, hence $a_{i i}=0$ and $b_{i i}=\sum_{k} w_{k i}^{2}+\operatorname{tr}\left(\mathbf{W}^{2}\right) / n$. Therefore, $\nu_{i}(\widehat{A P L E}, \mathbf{Z})=0$ in that case for any $n$.

\section{Applications}

\subsection{Pollution Data}

We consider the application of our methodology to the analysis of pollution levels arising from the pumping of waste material into the English Channel and reported by Haining (1990, p. 217). The dataset consists of reflectance values extracted from an aerial survey and located on a regular $9 \times 9$ spatial lattice. High levels of pollution induce high reflectance values. Following a previous study of this pollution data by Haining (1987), a linear trend in the reflectance values is first removed, yielding residuals $Z\left(s_{1}, s_{2}\right), s_{1}, s_{2}=1, \ldots, 9$. Our interest is now in modeling the possible dependence structure in these residual values. To this end, we compute the empirical variogram $2 \widehat{\gamma}(h)$ at spatial lag distances $h=1,2,3,4$ assuming isotropy.

Genton and Ronchetti (2003) have noticed several possible outliers in the residual values at locations $(1,1),(1,2),(2,2),(2,7)$ and $(7,5)$ on the lattice, and the largest residual takes the value 40 at location $(2,2)$. For this reason, we investigate the influence of each residual 
on the variogram estimator. Figure 2 depicts hair-plots of the sample variogram $2 \widehat{\gamma}(h)$ on the reflectance residual values for $h=1,2,3,4$ and $\zeta \in[-40,40]$. The variogram estimates on the original data at each lag $h$ are identified by a closed black disc. The influence of the largest residual (the observation \#17) is identified by the black curve. Notice that the influence changes from one lag to another. For example for $\zeta>0$, the observation \#17 is

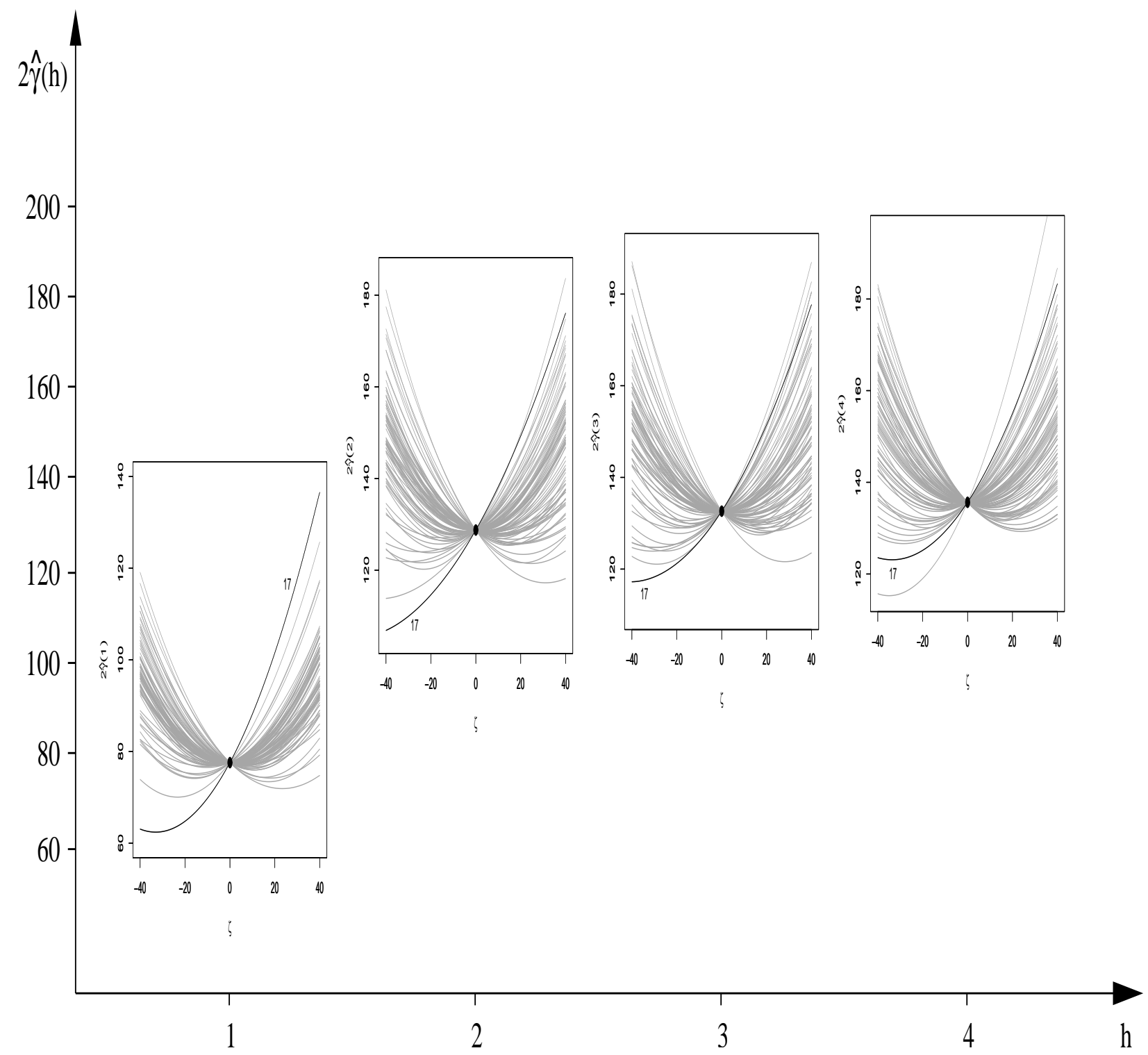

Figure 2: Hair-plots of the sample variogram $2 \widehat{\gamma}(h)$ on the reflectance residual values for spatial lag distance $h=1,2,3,4$. 
the most influential at lag $h=1$, but not at other lags. This fact has been the motivation of Genton (1998b) for the definition of a spatial breakdown-point of variogram estimators; see also Lark (2008) for recent discussions on this topic. In addition, as can be seen in Figure 2,
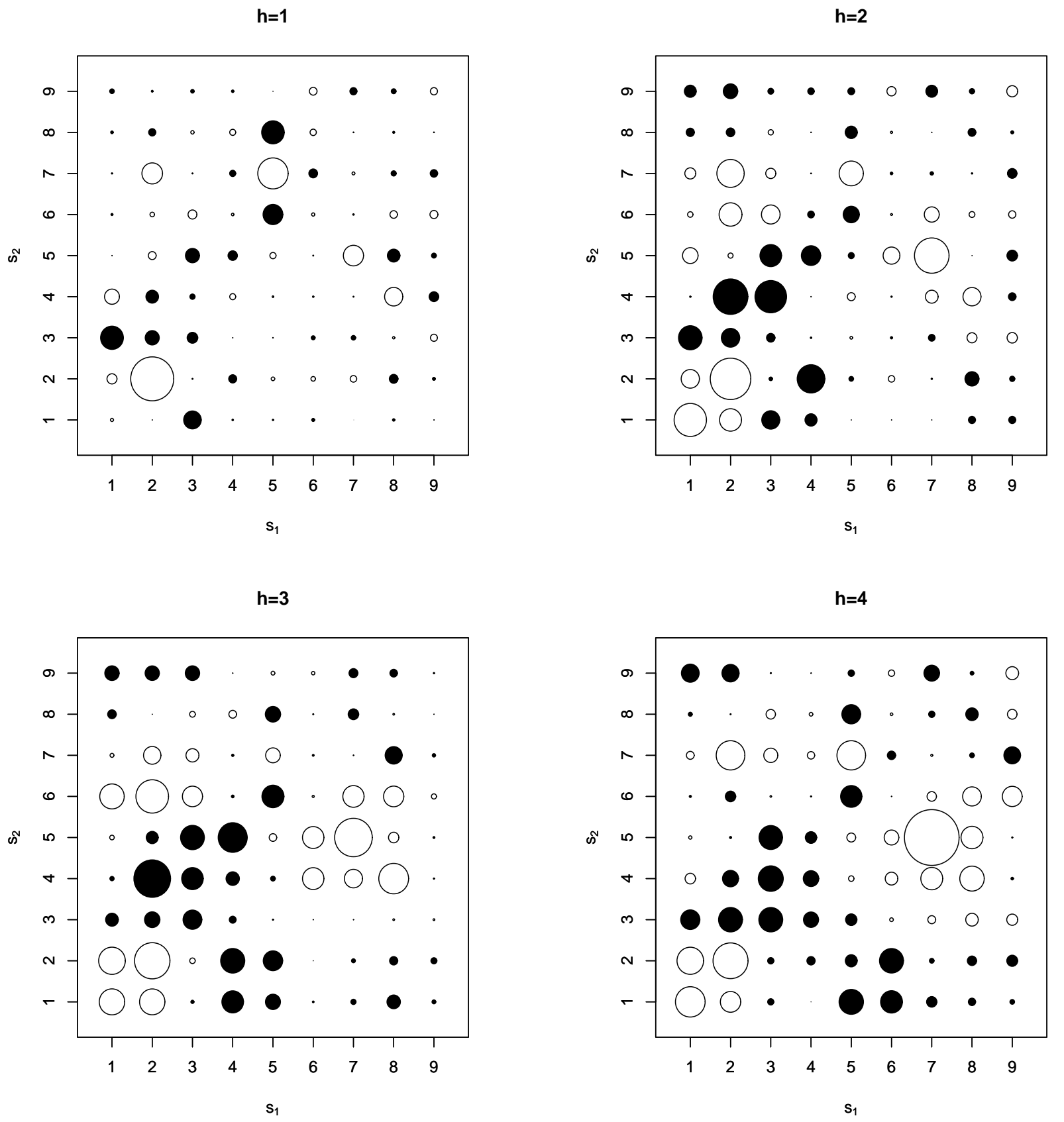

Figure 3: Disc-plot for $\zeta>0$ of the sample variogram $2 \widehat{\gamma}(h)$ on the reflectance residual values for lag distances $h=1,2,3,4$. 
the effect of a perturbation $\zeta$ results in a quadratic departure.

The influence of each observation can be depicted spatially. Figure 3 presents a discplot for $\zeta>0$ of the sample variogram $2 \widehat{\gamma}(h)$ on the reflectance residual values for each $h=1,2,3,4$. The radii of the discs are proportional to the rate of departure from $2 \widehat{\gamma}(h)$ due to a small contamination at each observation. Open discs denote an increase of the value

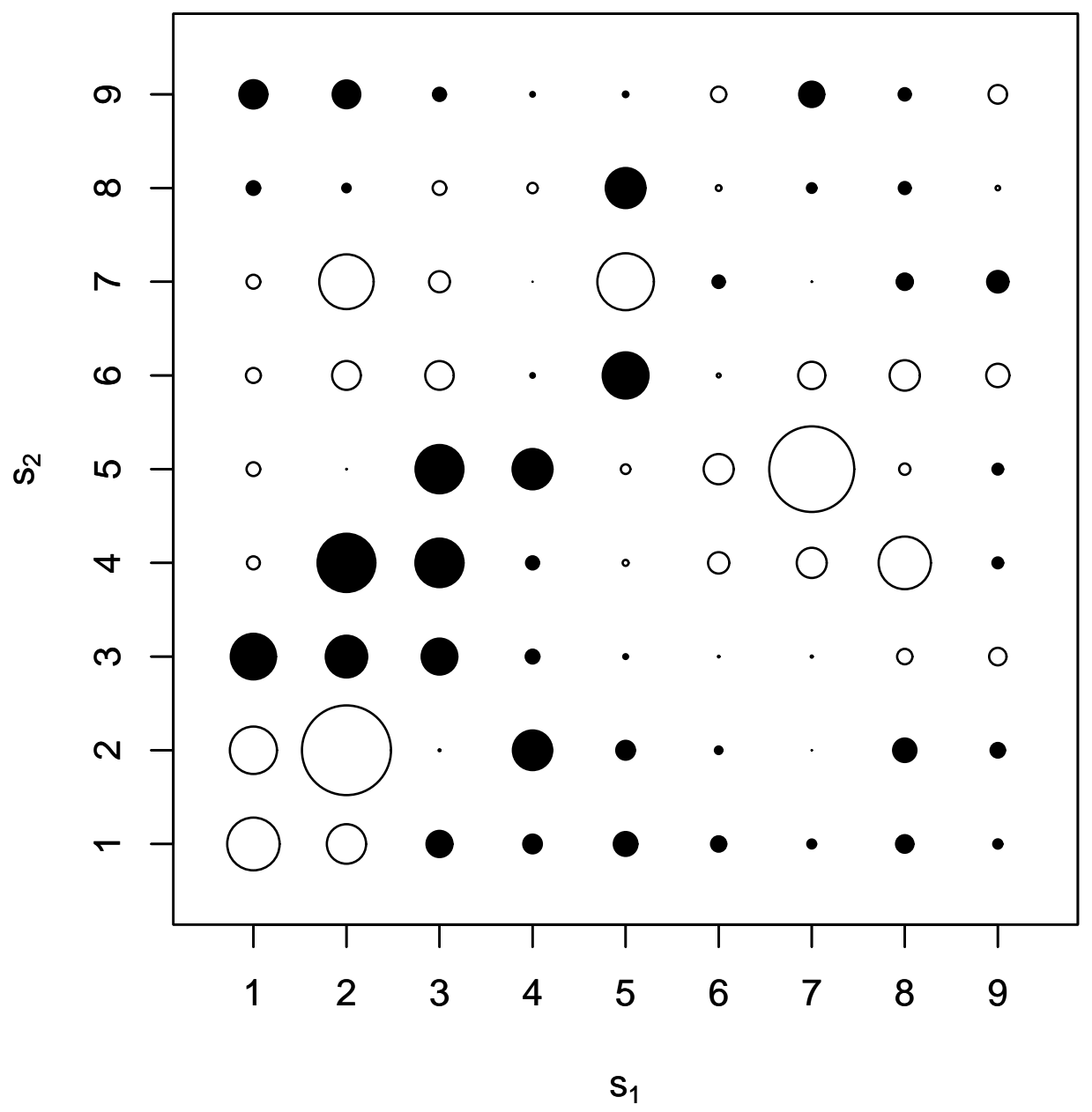

Figure 4: Disc-plot for $\zeta>0$ of the sample variogram $2 \widehat{\gamma}(h)$ on the reflectance residual values, averaged over lag distances $h=1,2,3,4$. 
$2 \widehat{\gamma}(h)$ whereas closed discs denote a decrease. The discs with large radii correspond to the most influential observations. Here also we can see that the influence changes from one lag to another. However, the observation \#17, located at $(2,2)$, is overall influential. In fact, the influence can be summarized over the various lags, for instance with a mean influence. This information is presented in Figure 4.

Note that when the locations of the observations are irregularly spaced, then the form of the matrix $\mathbf{A}$ in the quadratic form defining the sample variogram estimator becomes more complex. In that situation, the visualization of influential observations by means of the hair-plot is even more useful.

\subsection{African Conflict Data}

The second application we consider is from the field of Economics. The dataset consists of a standardized measure of total conflict for 42 African countries. It was used by Anselin (1995) in order to study the importance of spatial effects in the statistical analysis of international conflicts. Following Anselin (1995), we consider a $W$-coding spatial structure matrix W based on the first-order contiguity (common border) in order to calculate the Moran index. For this conflict measure, Moran's I equals 0.417 and strongly evidences positive spatial autocorrelation. Figure 5 displays the hair-plot of this autocorrelation index with four countries identified by black curves and labels. South Africa, and to a minor extent Senegal, are among the most influential units for any $\zeta \in[-10,10]$. More precisely, the hair-plot reveals that a positive contamination $\zeta$, i.e., a larger total conflict measure, for these countries leads to a rapid decrease of the autocorrelation index while a low negative contamination leads to an increase of the index. Sudan is also particularly influential when considering high negative $\zeta$ values leading to a negative Moran index. Finally, Egypt is not very influential when considering a low level of contamination $\zeta$ (positive or negative) but the change in 


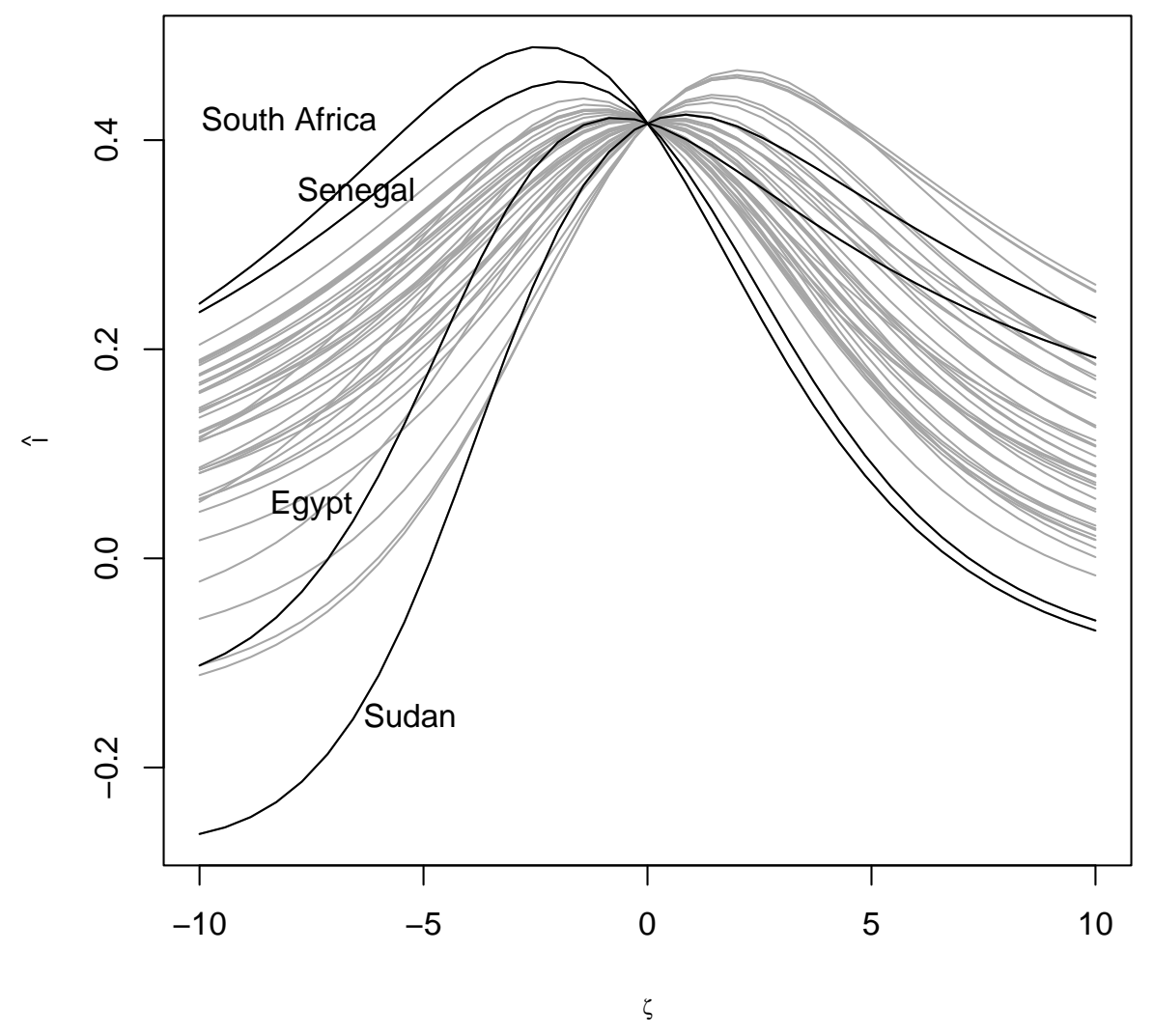

Figure 5: Hair-plot of Moran's $I$ estimator $\widehat{I}$ for the African conflict data.

the Moran index for large negative $\zeta$ is very steep and noticeable. Unlike in the first application, the effect of $\zeta$ is now a ratio of two quadratics. Furthermore, depending on the specific observation, the maximum of the curve is shifted to the left or to the right leading to different influential behaviors. The asymptotic influence of the $i$-th African country is $\nu_{i}(\widehat{I}, \mathbf{Z})=-w_{i} / 41$ and ranges from -0.066 (South Africa) to -0.003 (Lesotho).

Figure 6 displays a disc-plot of the Moran index overlayed on the Africa map. As in the previous application, open discs denote an increase of the value of Moran's $I$, whereas closed discs denote a decrease. The discs with large radii correspond to the most influential observations. We recover some of the previous results, namely that South Africa and Senegal 


\section{Local Sensitivity to Positive Contamination}

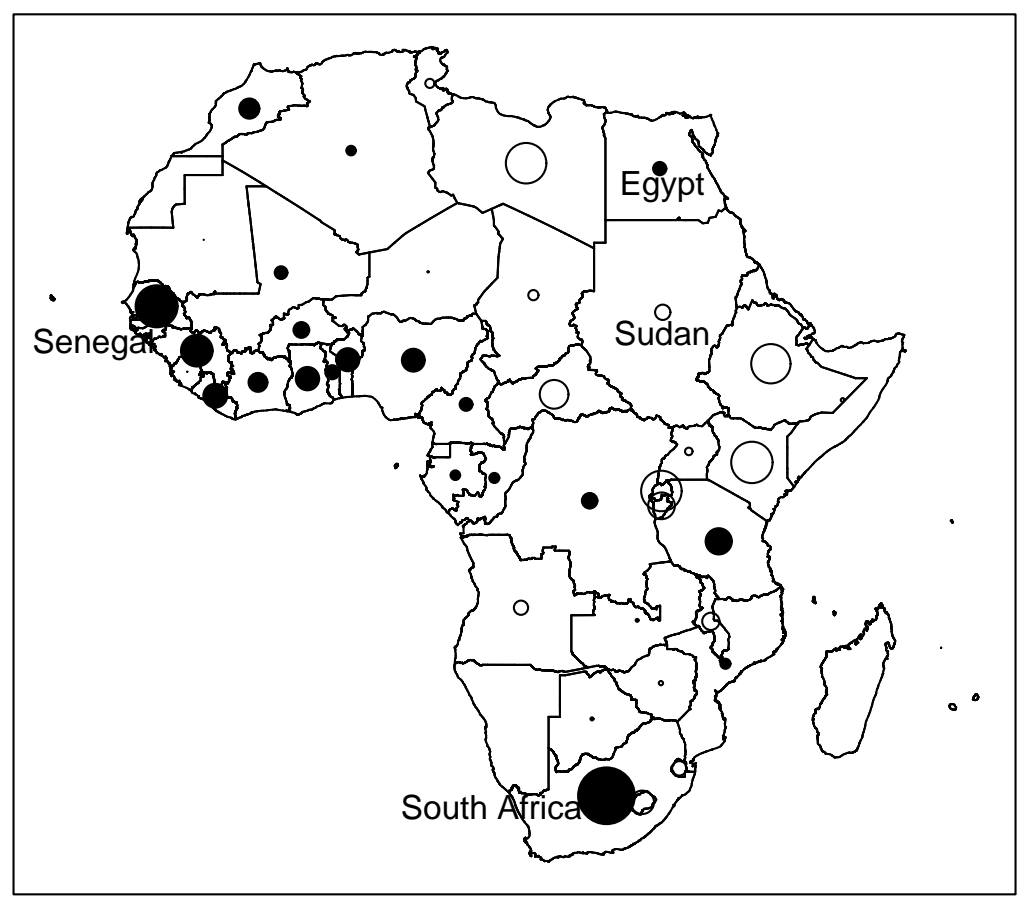

Figure 6: Disc-plot $(\zeta>0)$ of Moran's $I$ estimator $\widehat{I}$ for the African conflict data.

are locally influential for positive contamination while Sudan and Egypt are not influential in that sense. Other countries that have not been selected on the hair-plot are associated with large open discs and could also be considered as influential observations.

\section{Discussion}

Many simple and natural estimators arising in the context of dependent data are unfortunately sensitive to perturbations of a single observation as we illustrated in this paper. This motivates the need for developing robust estimators for time series and spatial data, and a few proposals can be found in the literature. Although classical estimators can often be 
defined as quadratic forms or ratios of quadratic forms in the data vector, robust estimators are typically more involved and therefore closed-form expressions for their local and asymptotic influence are not available. In that case, the hair-plot becomes very useful as it allows to visualize the sensitivity of each observation on complex estimators.

For instance, Ma and Genton (2000) have proposed a highly robust autocorrelation estimator

$$
\widehat{r}_{H R}(h)=\frac{Q_{n-h}^{2}(\mathbf{U}+\mathbf{V})-Q_{n-h}^{2}(\mathbf{U}-\mathbf{V})}{Q_{n-h}^{2}(\mathbf{U}+\mathbf{V})+Q_{n-h}^{2}(\mathbf{U}-\mathbf{V})}, \quad 0 \leq h \leq n-1,
$$

where $Q_{n-h}$ is a highly robust scale estimator of a sample of size $n-h$ proposed by Rousseeuw and Croux (1993), and $\mathbf{U}$ and $\mathbf{V}$ represent the first and last $n-h$ observations of the data vector $\mathbf{Z}$, respectively. We apply this highly robust autocorrelation estimator to the Austrian

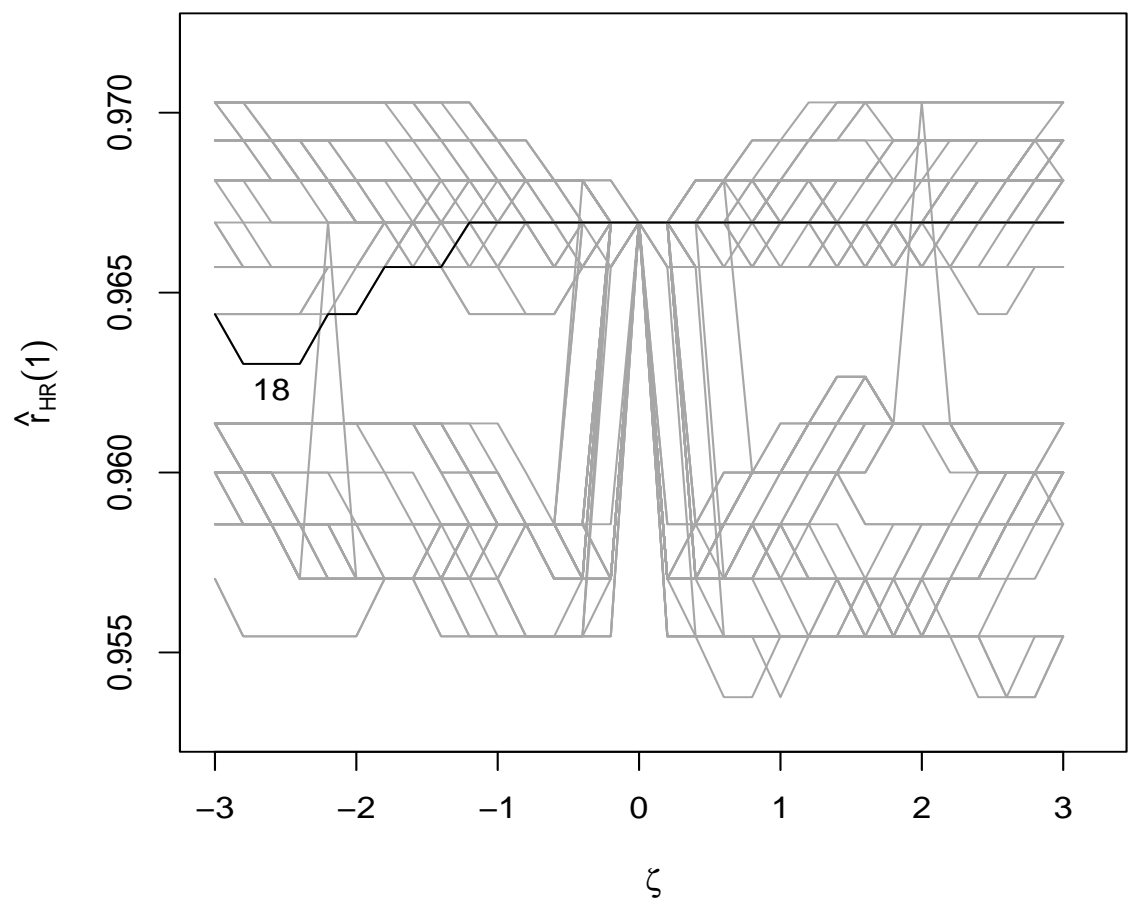

Figure 7: Hair-plot of the highly robust lag-one autocorrelation estimator $\widehat{r}_{H R}(1)$ on the Austrian bank monthly interest rates dataset. 
bank monthly interest rate data and present the associated hair-plot for $\widehat{r}_{H R}(1)=0.966$ in Figure 7. This value is in line with the optimal robust estimate of 0.96 obtained by Künsch (1984), with the value of 0.98 obtained by Azzalini and Genton (2008) based on a skew- $t$ distributional assumption, and with the range of values 0.89-0.96 in Wang et al. (2009). In Figure 7, the patterns of the curves are mainly due to the discreteness of the sample quantiles involved in the computation of $Q_{n-h}$, but overall the vertical variability is very small and the observation for the month 18 is no longer influential on this highly robust autocorrelation estimator.

In this paper we have focused on the influential effect of a single observation at a time for computational and visual simplicity. However, the influence of multiple observations, $k>1$ say, could be studied as well, each with possibly different contamination magnitudes $\zeta_{1}, \ldots, \zeta_{k}$. Clearly, this would lead to an explosion of the number of hairs ( $n$ choose $k$ ) and to dimensional difficulties in their graphical representations. Nevertheless, a particular case of interest is when those $k$ magnitudes are all equal, to $\zeta$ say. Then, for an estimator defined as a ratio of two quadratic forms in the data vector as in Section 3, the asymptotic influence of $k$ observations with indices in the set $\mathcal{I}$ is given by:

$$
\nu_{\mathcal{I}}\left(\widehat{\theta}_{R Q}, \mathbf{Z}\right)=\frac{\sum_{i, j \in \mathcal{I}} a_{i j}}{\sum_{i, j \in \mathcal{I}} b_{i j}} .
$$

Clearly, $\nu_{\mathcal{I}}$ depends on the set $\mathcal{I}$ and can be different from the simpler case given by $k=1$ that we studied earlier.

For illustration, we return to the time series of monthly interest rates of an Austrian bank. We had noticed three possible outliers at months 18, 28 and 29 of about the same magnitude. The influence of those $k=3$ observations on the lag-one sample autocorrelation $\widehat{r}(1)$ as a function of the common contamination $\zeta \in[-40,40]$ is represented by the dashed curve in Figure 8. Notice that the dashed curve has a maximum for $\zeta$ around -2 , which corresponds to bringing the three outlying observations down to the bulk of the data, see Figure 1 . In 


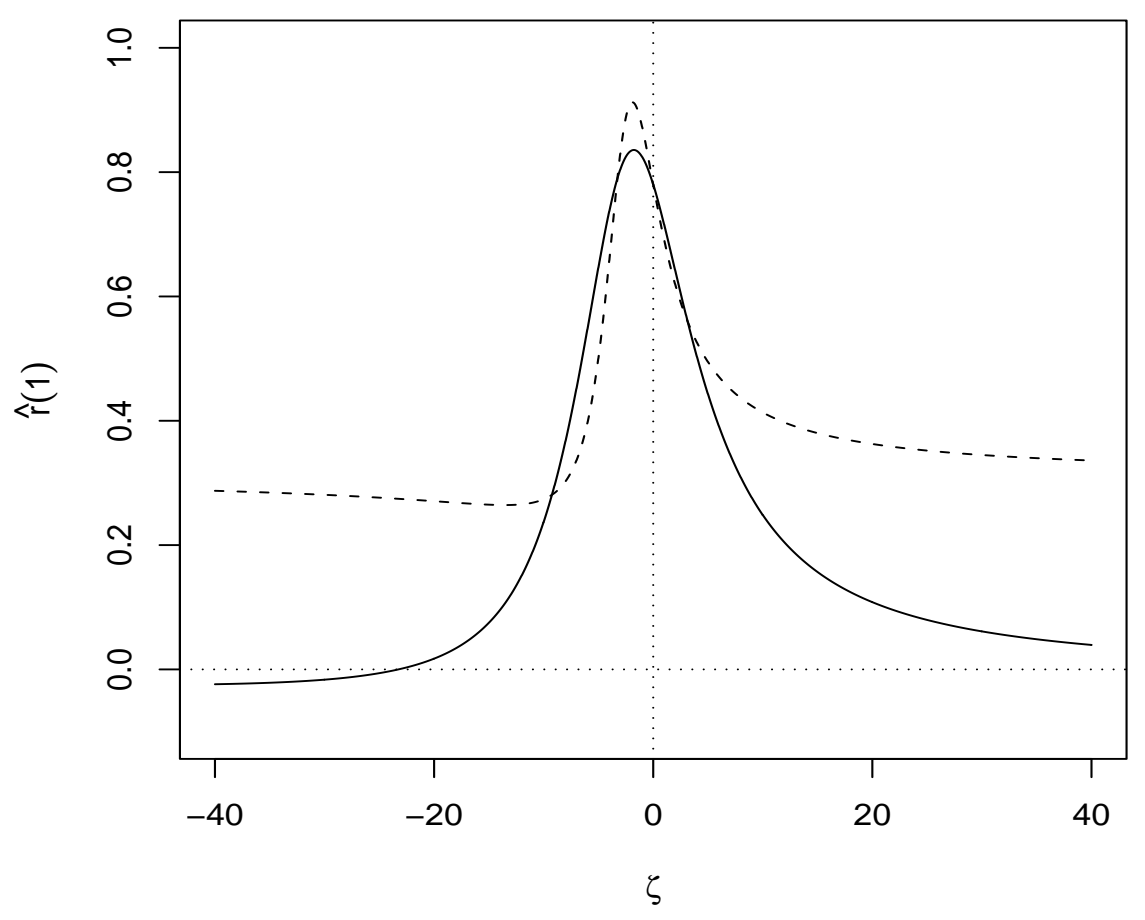

Figure 8: Hair-plot based on various contaminated observations of the lag-one sample autocorrelation $\widehat{r}(1)$ on the monthly interest rates dataset: month 18 contaminated (solid curve) and months 18, 28, 29 contaminated (dashed curve).

that case the value of $\widehat{r}(1)$ increases, as expected from the highly robust autocorrelation estimator above. From (10), the corresponding asymptotic influence for $\mathcal{I}=\{18,28,29\}$ is given by:

$$
\nu_{\mathcal{I}}(\widehat{r}(1), \mathbf{Z})=\frac{n^{2}-9 n-9}{3 n(n-3)}
$$

and takes the value 0.310 for the sample size $n=91$. For comparison, the influence of the sole observation at month 18 on the lag-one sample autocorrelation $\widehat{r}(1)$ is represented by the solid curve in Figure 8. From (6), its corresponding asymptotic influence is given by:

$$
\nu_{18}(\widehat{r}(1), \mathbf{Z})=\frac{-n-1}{n(n-1)}
$$

and takes the value -0.011 for the sample size $n=91$. Hence, those two asymptotic 
influences are different, as can be seen in Figure 8.

\section{Appendix}

\section{Proof of Proposition 1}

By assumption:

$$
f\left(y_{1}, y_{2}+\zeta, y_{3}, \ldots, y_{n}\right)=f\left(y_{1}+\zeta, y_{2}, y_{3}, \ldots, y_{n}\right)
$$

for any $y_{1}, \ldots, y_{n}$. Consequently, for $y_{1}=z_{1}, y_{2}=0, y_{i}=z_{i}, i=3, \ldots, n$, and $\zeta=z_{2}$, we have:

$$
f\left(z_{1}, z_{2}, z_{3}, \ldots, z_{n}\right)=f\left(z_{1}+z_{2}, 0, z_{3}, \ldots, z_{n}\right)
$$

and by recurrence,

$$
f\left(z_{1}, z_{2}, z_{3}, \ldots, z_{n}\right)=f\left(\sum_{i=1}^{n} z_{i}, 0,0, \ldots, 0\right)=g\left(\sum_{i=1}^{n} z_{i}\right),
$$

a function $g$ of $\sum_{i=1}^{n} z_{i}$ only. This concludes the proof. 


\section{References}

Anselin, L. (1995), "Local indicators of spatial association-LISA," Geographical Analysis, $27,93-115$.

Azzalini, A., and Genton, M. G. (2008), "Robust likelihood methods based on the skew- $t$ and related distributions," International Statistical Review, 76, 106-129.

Cliff, A. D., and Ord, J. K. (1981), Spatial Processes: Models and Applications, London: Pion.

Cressie, A. C. (1993), Statistics for Spatial Data, 2nd edition, New York: J. Wiley \& Sons.

Genton, M. G. (1998a), "Variogram fitting by generalized least squares using an explicit formula for the covariance structure," Mathematical Geology, 30, 323-345.

Genton, M. G. (1998b), "Spatial breakdown point of variogram estimators," Mathematical Geology, 30, 853-871.

Genton, M. G. (1999), "The correlation structure of the sample autocovariance function for a particular class of time series with elliptically contoured distribution," Statistics and Probability Letters, 41, 131-137.

Genton, M. G. (2003), "Breakdown-point for spatially and temporally correlated observations," in Developments in Robust Statistics, International Conference on Robust Statistics 2001, R. Dutter, P. Filzmoser, U. Gather, and P. J. Rousseeuw (eds), Springer, Heidelberg, 148-159.

Genton, M. G., and Lucas, A. (2003), "Comprehensive definitions of breakdown-point for independent and dependent observations," Journal of the Royal Statistical Society, Series $B, 65,81-94$.

Genton, M. G., and Lucas, A. (2005), discussion of "Breakdown and groups" by L. Davies and U. Gather, Annals of Statistics, 33, 988-993.

Genton, M. G., and Ronchetti, E. (2003), "Robust indirect inference," Journal of the American Statistical Association, 98, 67-76.

Gorsich, D. J., Genton, M. G., and Strang, G. (2002), "Eigenstructures of spatial design matrices," Journal of Multivariate Analysis, 80, 138-165. 
Hampel, F. R., Ronchetti, E. M., Rousseeuw, P. J., and Stahel, W. A. (1986), Robust Statistics. The Approach Based on Influence Functions, New York: Wiley.

Haining, R. (1987), "Trend-surface models with regional and local scales of variation with an application to aerial survey data," Technometrics, 29, 461-469.

Haining, R. (1990), Spatial Data Analysis in the Social and Environmental Sciences, Cambridge, U.K.: Cambridge University Press.

Hillier, G., and Martellosio, F. (2006), "Spatial design matrices and associated quadratic form: structure and properties," Journal of Multivariate Analysis, 97, 1-18.

Künsch, H. (1984), "Infinitesimal robustness for autoregressive processes," Annals of Statistics, 12, 843-863.

Lark, R. M. (2008), "Some results on the spatial breakdown point of robust point estimates of the variogram," Mathematical Geoscience, 40, 729-751.

Li, H., Calder, C., and Cressie, A. C. (2007), "Beyond Moran's I: Testing for spatial dependence based on the spatial autoregressive model," Geographical Analysis, 39, 357-375.

Ma, Y., and Genton, M. G. (2000), "Highly robust estimation of the autocovariance function," Journal of Time Series Analysis, 21, 663-684.

Moran, P. A. P. (1950), "Notes on continuous stochastic phenomena," Biometrika, 37, 17-23.

R Development Core Team (2009). R: A language and environment for statistical computing. R Foundation for Statistical Computing, Vienna, Austria. ISBN 3-900051-07-0, URL http://www.R-project.org.

Rousseeuw, P. J., and Croux, C. (1993), "Alternatives to the median absolute deviation," Journal of the American Statistical Association, 88, 1273-1283.

Tiefelsdorf, M. (2000), Modelling Spatial Processes - The Identification and Analysis of Spatial Relationships in Regression Residuals by Means of Moran's I, Lecture Notes in Earth Sciences 87, Berlin: Springer Verlag.

Wang, Q., Stefanski, L. A., Genton, M. G., and Boos, D. (2009), "Robust time series analysis via measurement error modeling," Statistica Sinica, 19, 1263-1280. 